\section{Combining FIB and Automated Microcleaving \\ Provides Fast, Accurate Cross Sections}

Janet Teshima, FEI Company \& Efrat Raz, SELA USA, Inc.

A large part of the preparation of semiconductor samples for SEM and TEM observations involves the creation of cross sections to expose subsurface defects and three-dimensional structure. A powerful new combination of FIB (FEI Company, Hillsboro, Oregon, http://www.feic.com ) with automated microcleaving technology (SELA, Santa Clara, California, http://www. sela.com ) now offers a comprehensive solution for fast, easy and accurate sample preparation.

\section{Cleave and Polish}

Traditionally cross sections have been prepared manually by cleaving the wafer along a crystal plane close to the desired site, then grinding and polishing the sample to reveal the feature of interest. This approach has several disadvantages. It is slow, typically requiring an hour or more for each preparation. It is difficult to perform accurately, especially for defects or features that are difficult to see in a light microscope. It requires a highly skilled technician. It is an inherently dirty process, prone to creating artifacts and smearing or obscuring fine detail. Finally, it is unreliable - the slightest misjudgement can easily destroy valuable information by polishing away the target.

\section{Focused Ion Beam}

In recent years FIB has become an important preparation tool for both SEM and TEM samples. Its most important benefits are the precise control it offers over cross section placement, and its ability to navigate to a desired location based on defect coordinates, CAD drawings or electrical test data. The speed and accuracy of automated microcleaving can improve FIB cross sectioning throughput by shortening the preparation time for the preliminary rough section and reducing the amount of material to be milled by the ion beam.

\section{Microcleaving}

Microcleaving is a fully automated technique that uses a precisely controlled diamond scribe, a tunable hammer, and a thorough understanding of the wafer's properties and composition to create accurate, high quality cross sections in a fraction of the time required by other techniques. In most cases it can place the plane of the cross section within less than a micron of the target location. The entire operation takes ten to fifteen minutes. The cross sections are clean and free of artifacts. Since no part of the sample is polished away, the feature of interest is

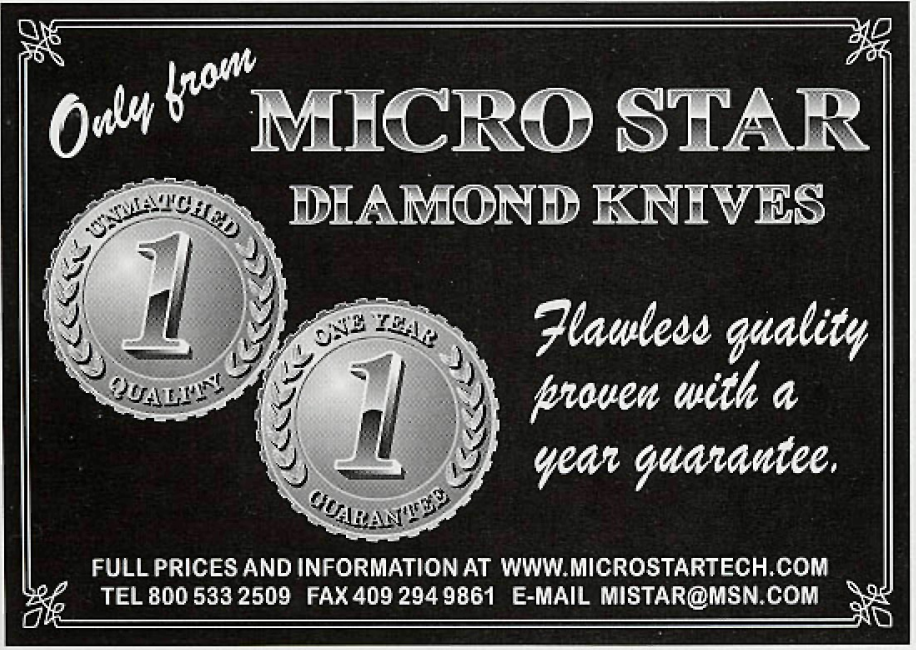

never destroyed. Even if the section misses the target, the target remains intact in one of the cleaved pieces.

\section{FIB and Microcleave}

Microcleaving alone does require the ability to see the target in an optical microscope. With today's shrinking geometries, some targets are difficult or impossible to see or may require better placement accuracy than the microcleaving system can provide. Here lies the power of combining microcleaving with FIB. Let's look at a few examples:

Microcleave then FIB-If the cleaving operation misses the target, the FIB can quickly remove the small amount of material still concealing the feature of interest. The operator may intentionally choose this approach when the location of the target is uncertain or to obtain a sequence of cross sections through a large target.

FIB then Microcleave-In some cases the target is not visible, for example, subsurface defects, or blind coordinates from a defect detector, electrical tester or CAD design. With its highaccuracy navigation the FIB can locate and cross section the targets. Subsequent microcleaving through the now visible FIB marks fully exposes the cross sections to normal incidence SEM/ EDS examination. Both the FIB and the microcleaving systems can accommodate multiple targets on a single wafer.

Saw and glue then FIB for TEM-A specially designed TEM sample preparation system can automatically cut a twenty micron thin cross section and glue it to a TEM sample grid-ready for final thinning to electron transparency in the FIB. The complete operation up to FIB readiness takes twenty minutes.

Together FIB and Microcleaving offer an effective solution to almost any conceivable cross-sectioning need. Their high throughput, low consumables, and minimal requirements for operator skill offer immediate and continuing savings in operating costs. The high accuracy, quality and reliability of the crosssectioning process guarantee the availability of critical information when it is needed most.
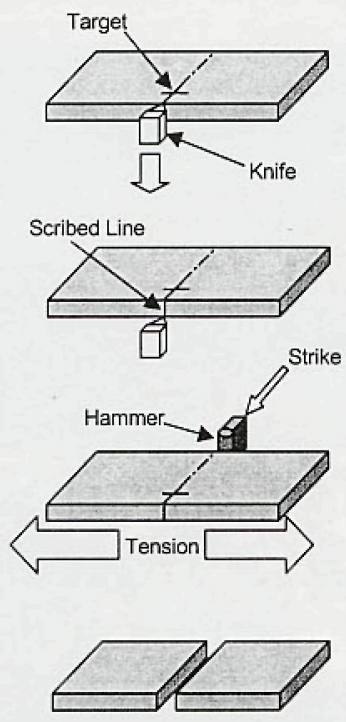

The automated microcleaving system can cleave targeted features with submicron accuracy. The user designates the target in an optical microscope. The system then automatically scribes the wafer edge in a location that will direct the cleaved plane through the target. It then applies tension to the wafer and strikes the edge opposite the scribe line to initiate the cleavage. 


\section{Remove the Veil of Contamination from SEM Samples!}

\section{Clean when contamination starts!}

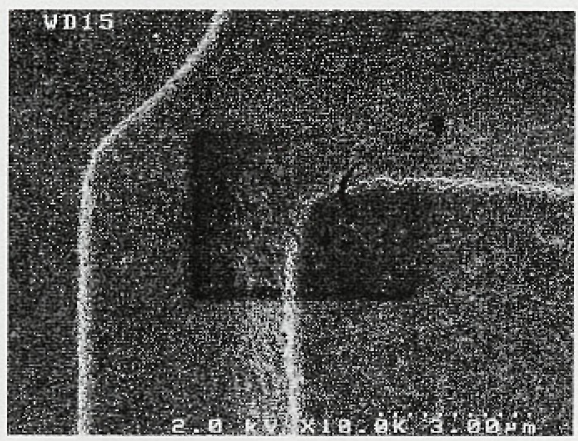

A contamination deposit was formed by a short SEM scan on a dirty specimen.

The Flash SEM-CLEAN process removed most of the deposit in 2 minutes and prevented more deposition. -Rick Passey HP

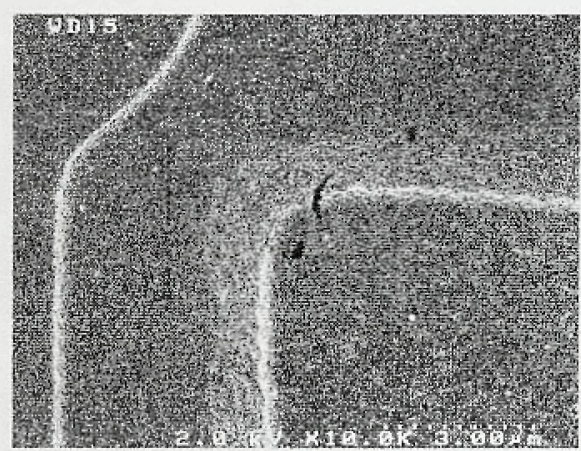

- Quickly remove hydrocarbon scum from SEM chamber walls and samples

- Clean samples in-situ

- Fast - cleans in minutes, not months

- Oxygen radical cleaning action

- Low power RF plasma radical source

- Compact - mounts on a chamber port

- Ideal for Materials Science and Metrology

- No exotic gases - No Argon

- Keeps detector windows clean

- Patent pending

\section{XEI scientific}

Anti-Contamination Systems for SEMs

3124 Wessex Way, Redwood City, CA 94061 (650) 369-0133, FAX (650) 363-1659

http//www. msa microscopy com/SM/XEI/XEIHomePage.html

\section{NEW! \\ Flash SEM-CLEAN in-situ Cleaning}

CD SEM Metrology precision improved:
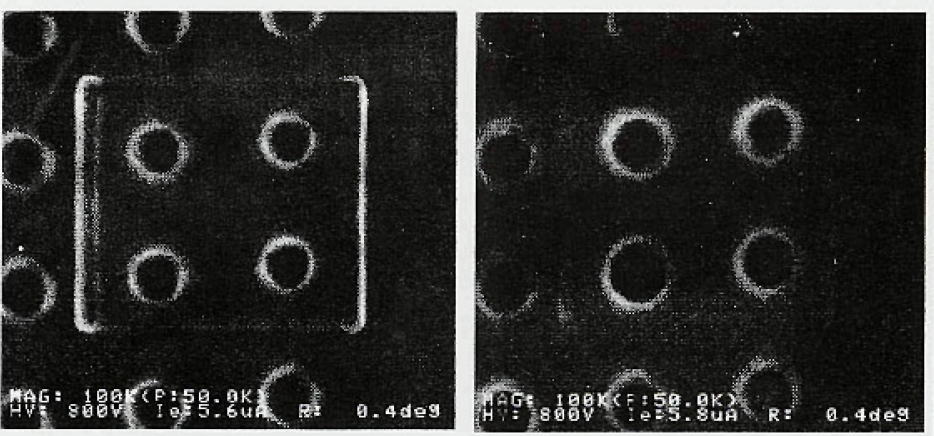

Before

After

A photoresist test pattern shows contamination filling in the holes before cleaning. Holes remain constant size after cleaning. Courtesy Andras Vladar-NIST

\section{Typical Installation:}

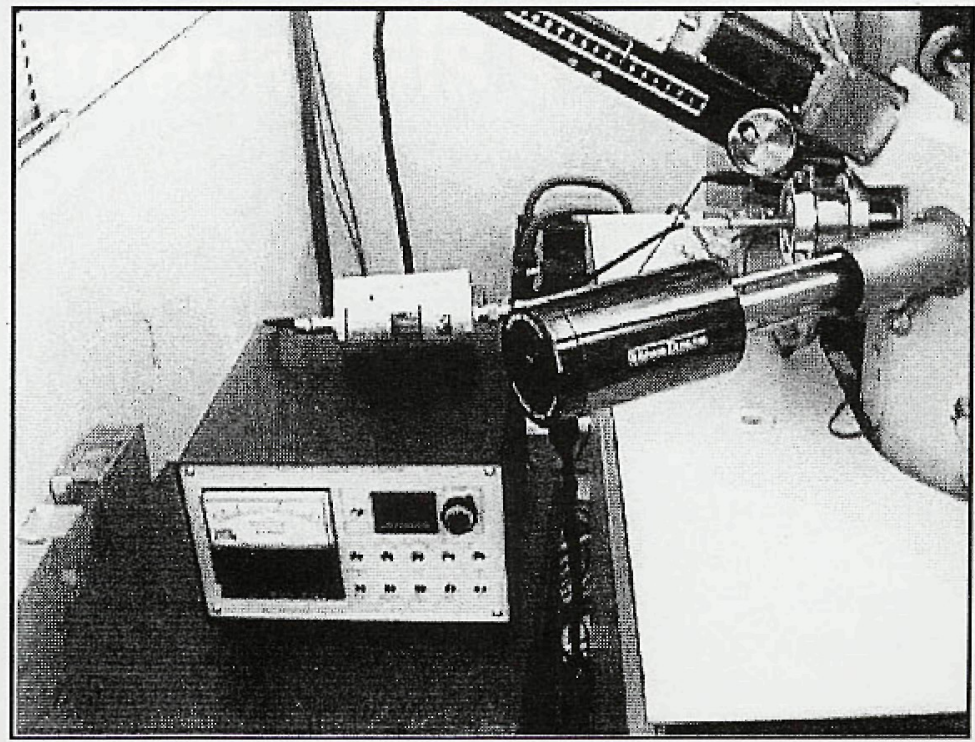

Compact Flash SEM-CLEAN system installs easily on crowded SEM ports. Shown above are the controller, RF match, and plasma head mounted between the backscatter detector and EDS. Courtesy Sharon Myers - IBM

\section{Flash SEM-CLEAN Removes the Oil!}

